\title{
Clindamycin Palmitate Hydrochloride
}

National Cancer Institute

\section{Source}

National Cancer Institute. Clindamycin Palmitate Hydrochloride. NCI Thesaurus. Code C61683.

The palmitate hydrochloride form of clindamycin, a semi-synthetic, chlorinated broad spectrum antibiotic produced by chemical modification of lincomycin. Clindamycin palmitate hydrochloride is used for oral suspension. 